\title{
An Allele-Specific Genotyping Method for Rat Lyst (Lysosomal Trafficking Regulator) Gene
}

\author{
Norio MASUI 1), Masayuki MORI2), Tetsu NISHIKAWA ${ }^{3)}$, Yumie TAKAGI1), \\ Hidekazu ASAI 1), Makoto YANABE ${ }^{1)}$, Ken-ichi YAMASAKI4), \\ Tadao SERIKAWA4), and Katsunori SATO5)
}

\begin{abstract}
1)Japan SLC, Inc., 3-5-1 Aoihigashi, Hamamatsu, Shizuoka 433-8114, 2)Department of Aging Biology, Institute for Aging and Adaptation, Shinshu University Graduate School of Medicine, 3-1-1 Asahi, Matsumoto, 390-8621, ${ }^{3}$ Institute for Experimental Animals, Hamamatsu University School of Medicine, 1-20-1 Handa-yama, Hamamatsu, Shizuoka 431-3192, ${ }^{4}$ Institute of Laboratory Animals, Graduate School of Medicine, Kyoto University, Sakyo-Ku, Kyoto 606-8501, and ${ }^{5)}$ Faculty of Agriculture, Okayama University, 1-1-1 Tsushimanaka, Okayama, 700-8530, Japan
\end{abstract}

\begin{abstract}
Two spontaneous mutant beige rats, with phenotypes resembling human Chediak- Higashi syndrome (CHS), were found independently in two inbred strains. Both beige mutations were identified to be recessive alleles in the Lyst locus on rat chromosome 17 and the alleles were denoted Lystbg and Lystbg-Kyo. As it is almost impossible to discriminate these mutations phenotypically, we developed an allele-specific genotyping method for the Lyst gene. The nested PCR amplification was followed by restriction fragment length polymorphism (RFLP) analysis. By this method, we could discriminate the mutant Lyst ${ }^{\mathrm{bg}}$, Lyst ${ }^{\mathrm{bg}-K y o}$ alleles, and the normal Lyst allele, easily and accurately.
\end{abstract}

Key words: Lyst alleles, nested PCR-RFLP analysis

Human Chediak-Higashi syndrome (CHS) is a rare autosomal recessive genetic disorder characterized by partial oculocutaneous albinism, mild predisposition to pyogenic infections, and giant granules in various cells $[2,3,14]$. Increased susceptibility of human CHS patients to infection may be explained by defects observed in T-cell cytotoxicity and natural killer (NK) cell activity as well as in chemotaxis and bacteriocidal capacity of granulocytes and monocytes $[1,5,7,15]$.

Two spontaneous beige rat mutations were found occurring independently. One was found in 1989 in an inbred colony of DA/Ham strain rats maintained at
Hamamatsu University School of Medicine [13]. Another was found in 1999 in an ACI/N rat colony maintained at the Institute of Laboratory Animals, Graduate School of Medicine, Kyoto University [10]. The genetic and phenotypic characteristics of these beige mutant rats were investigated and both mutants were regarded as useful animal models for human CHS. Both beige mutations resulted from a genomic deletion of about $20 \mathrm{~kb}$ containing exons 28,29 , and 30 , which constitute the 578-bp sequence missing in the Lyst cDNA on rat chromosome (Chr) 17 [8, 9, 12]. Each deletion junction was formed by a recombination be- 
tween the different sites of the same two Long Interspersed Nucleolar Element 1s (L1) within the Lyst gene [10]. Accordingly, these Lyst mutations were demonstrated to be alleles of the Lyst locus and were denoted $L y s t^{b g}$ and $L y s t^{b g-K y o}$. However it is almost impossible to discriminate between the Lyst $t^{b g}$ allele and the Lyst ${ }^{b g-K y o}$ allele phenotypically, except that each beige mutant comes from a different genetic background. We developed a method for discriminating the rat Lyst alleles and a simple genotyping method for the rat Lyst locus.

Firstly, female DA/Slc rats were mated with male DA/Ham-Lyst $t^{b g} / L y s t^{b g}$ homozygotes at 8 weeks of age and thirteen progenies were obtained in all. Similarly, female ACI/N rats were mated with male ACI/NLyst $t^{\text {b-Kyo }} /$ Lyst ${ }^{\text {bg-Kyo }}$ homozygotes. Using standard techniques with phenol- $\mathrm{CHCl}_{3}$, the genomic DNA was isolated from the tissue of a tailpiece taken out from each progeny and their parents.

Three pairs of primers $\mathrm{Bg} 41 \mathrm{C} / / \mathrm{Bg} 50, \mathrm{Bg} 39 \mathrm{~A} 4 / /$ $\mathrm{Bg} 52 \mathrm{~A} 4$, and $\mathrm{Bg} 39 \mathrm{~A} 4 \mathrm{nes} / / \mathrm{Bg} 52 \mathrm{~A} 4$ nes were synthesized for the genotyping of the Lyst locus (BEX CO., LTD). The $\mathrm{Bg} 41 \mathrm{C} / / \mathrm{Bg} 50$ primer pair was designed based on the nucleotide sequence of the mouse, and rat Lyst cDNA was used for detecting the normal Lyst allele. Each primer sequence was as follows: Bg41C, 5'-CGGC TGCAGAGGCTCACCATCTTAGCTGTGAACA-3' (nucleotide positions 7885 to 7918 of GenBank AB020019 deposit), and Bg50, 5'-GAGGTCTT GCTTTGGGATGT-3' (nucleotide positions 7978 to 7997 of GenBank AB020019 deposit) [9, 11]. The $\mathrm{PCR}$ analysis by the $\mathrm{Bg} 41 \mathrm{C} / / \mathrm{Bg} 50$ primer pair was carried out with initial incubation at $94^{\circ} \mathrm{C}$ for $5 \mathrm{~min}$, followed by 30 cycles of denaturation at $94^{\circ} \mathrm{C}$ for $30 \mathrm{~s}$, annealing at $60^{\circ} \mathrm{C}$ for $30 \mathrm{~s}$, and extension at $68^{\circ} \mathrm{C}$ for 4 min, with a final extension at $72^{\circ} \mathrm{C}$ for $7 \mathrm{~min}$. The $\mathrm{Bg} 39 \mathrm{~A} 4 / / \mathrm{Bg} 52 \mathrm{~A} 4$ and the Bg39A4nes//Bg52A4nes primer pairs were used for detecting the mutant $L y s t^{b g}$ and Lyst ${ }^{\text {bg-Kyo }}$ alleles. The Bg39A4//Bg52A4 primer pair was designed based on the cDNA sequence of the rat Lyst gene and each primer sequence was as follows: Bg39A4, 5'-CTACCACCATTCAGTGCCTCAGAA GAGGAGAAGCATTGC-3' (nucleotide positions 7701 to 7739 of GenBank AB020019 deposit), and Bg52A4, 5'-ATCAGCTCTGACAAATACAAAACCAACTT GGCTCC-3' (nucleotide positions 8323 to 8357 of GenBank AB020019 deposit) [16]. The Bg39A4nes// $\mathrm{Bg} 52 \mathrm{~A} 4 \mathrm{nes}$, which is the inner primer pair for the nested

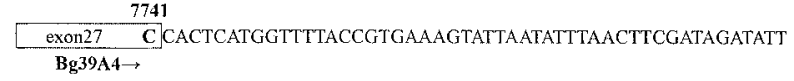

TAAGGACATAAGACTACGGTAAATATAATTTTTATAAAAATAACGACTCGTATCTCCGTAC ACATGACCCCCTTGTTTCCGTTCATACAAAGCCCTCAAACTCCAGTCGGACCAGATCGTA TGACTTAGAGAACAGAGGTCTTTGATTCCTTAATTTTGTAACAAGAACCAAATTTTTTTTA CCTTAATATTTTAGACTCCTTIAAAAGGAGGTCGTCTTTGGTAACATGGTCGTCTCAGATG TATCTGGTACAAGTGCAAAATAGGATTGCCTITAGTAGTTTCAAAGGTCCCCTACGTACTA TACTAGTCTATCTATAGACAACATTTCTCTCTTGGGACTGAAGACACCCCTTATACTTGAA CETCTTTGGTTTCACGTCTTGgetacatcetcgacacegtegtaptteatct ACACTATCNGAACATCTO Bg39Atnes $\rightarrow$

TCCGTTTTACNT $-\boldsymbol{b}-\boldsymbol{K}-$ exon 28 exon 29 exon $30-\boldsymbol{b}-\boldsymbol{K}$-TTGTTTCCCAGGTGT Bg41C $\rightarrow \rightarrow B g 50$

GTCTGCCCctetgaagetetagetetcecttceacgggatttGGGTGCAGGGAGCTGCTTGACTGGGTCCTT $4-B g 52 A 4 n e s$

TCAAATCCAGGTGCAGTCTGGACCACAGGGCTCCTGCAGCTTGACTGCTCCAATCTTCCT GTGCCCAGAGGCAGTATACAGACAGTTTCTCTTGGGCCAAGGATGTGGGCAAAGGTGGA CAGAAGTGACAGTCTCTCCTGCCCTGTAGTCTCAGGATTGCCCACACTTCTGGGCAATTA GCTCTCTCTCCCACTGGGTTTGGGAGCAGGGAGCTGTGGGCTGGGACAGCGAGGTTTGG GCGCCAGCTTCAAAACATCGTTTATATTACTTTTAAAAGTACTTTTTTCCTATTATTTAATA AAATTAGGAATAATTCTCTAATATCAAATACCTAATACATTATTAAATTTCTTCATCTAGTCT ATCATGTCTTTTATAGTTATATTAAATCTGACCCAATCAACTAACTTTAGAAATATATTTAAA TTATCATTAAATAATTTAAAATAAAAACAAATATAATTAAATTTATTATTAAATAATTTAAAT

GTAAGAAATAAAAATCTTGTAACTAAAGCCTTATTACTTATTTTTAAAG $\frac{\mathbf{8 3 1 9}}{\leftarrow \quad \text { exon 31 }}$

Fig. 1. Partial nucleotide sequence of genomic DNA amplified with the Bg39A4//Bg52A4 primer pair and the positions of the primer pairs used for the genotyping of the Lyst gene. White boxes represent exons. The numbers on the upsides of the boxes indicate the nucleotide positions on the rat Lyst cDNA. The recombination sites of the Lyst $t^{b}$ and the $L y s t^{b g-K y o}$ alleles are indicated by $b$ and $K$, respectively.

PCR analysis, was designed as follows. Using genomic DNA isolated from the DA/Ham-Lyst $t^{b g} / L y s t^{b g}$ homozygote, PCR was carried out with the Bg39A4//Bg52A4 primer pair. As a result of the amplification, a DNA fragment of about $13 \mathrm{~kb}$ was obtained. We sequenced about $1 \mathrm{~kb}$ of both ends of the genomic DNA fragment by the Dye terminator method from BEX CO., LTD (Fig. 1). The Bg39A4nes inner primer was designed within one of the sequenced regions and the Bg52A4nes inner primer was within the other sequenced region and each primer sequence was as follows: Bg39A4nes, 5'CCATGTAGGAGCTGTGGCAGCAATCAAGGTAGAC-3' and Bg52A4nes, 5'-AAATCCCGTGGAAGGGAGA GCTAGACCTTCAGAG-3'. For detecting both mutant alleles, the first round of PCR amplification by the Bg39A4//Bg52A4 primer pair was carried out with initial incubation at $94^{\circ} \mathrm{C}$ for $1 \mathrm{~min}$, followed by 25 cycles of denaturation at $94^{\circ} \mathrm{C}$ for $30 \mathrm{~s}$, annealing and extension at $68^{\circ} \mathrm{C}$ for 18 min with a final extension at $72^{\circ} \mathrm{C}$ for 10 min according to the manufacturer's instruction 
of LA Taq (TAKARA SHUZO CO., LTD). Each first PCR product was diluted one-tenth with distilled water and was used as a template in the subsequent round of the nested PCR. The second PCR amplification by the Bg39A4nes//Bg52Anes inner primer pair was carried out with the same conditions of the first PCR analysis. The PCR products were electrophoresed on $1 \%$ agarose gels (SeaPlaque GTG Agarose, Bio Whittaker Molecular Application, Inc.) in $1 \times$ TAE buffer and were stained with ethidium bromide.

Using PCR analysis by the $\mathrm{Bg} 41 \mathrm{C} / / \mathrm{Bg} 50$ primer pair, a DNA fragment of about $3.5 \mathrm{~kb}$ was amplified from ACI/N-Lyst $t^{b-K y o} /+$ heterozygotes, DA-Lyst $t^{b g} /+$ heterozygotes and their wild-type inbred strains (Fig. 2A), but this targeted PCR product was not obtained from ACI/ $\mathrm{N}-L y s t^{b g-K y o} / L y s t^{b g-K y o}$ homozygotes and DA/Ham-Lyst ${ }^{b g} /$ $L y s t^{b g}$ homozygotes. On the other hand, the nested PCR analysis by the Bg39A4//Bg52A4 and the Bg39A4nes//Bg52A4nes primer pairs amplified about $11 \mathrm{~kb}$ of the DNA fragment in ACI/N-Lyst $t^{b-K y o} /+$ heterozygotes, DA-Lyst $t^{b g} /+$ heterozygotes and their mutant homozygotes (Fig. 2B), while this targeted PCR product was not obtained from their normal wild-type inbred strains. On electrophoresis using agarose gel, however, the $11 \mathrm{~kb}$ DNA fragment amplified from the Lyst $t^{b g}$ allele was indistinguishable from that amplified from the Lyst $t^{b g-K y o}$ allele. The nested PCR product of each mutant allele was therefore digested with restriction endonucleases according to the respective manufacturer's instructions and each individual digest was electrophoresed on $1 \%$ agarose gel. The restriction endonucleases used for the RFLP analysis were as follows: BamHI, BglII, BseDI, BssHII, DdeI, EcoRI, EcoRV, MboI, MspI, SalI, StuI, TaqI, Tru9I, Tsp45I, and XbaI. Only the electrophoretic pattern of the StuI digests could discriminate between the Lyst ${ }^{b g}$ allele and the Lyst $t^{b-K y o}$ allele. In addition to two DNA fragment of about $5.5 \mathrm{~kb}$ and $0.3 \mathrm{~kb}$, which were equally observed in both mutant alleles, a DNA fragment of about $5 \mathrm{~kb}$ observed in the Lyst $t^{b g-K y o}$ allele was divided into two DNA fragments of about $4 \mathrm{~kb}$, and $0.7 \mathrm{~kb}$ in the Lyst ${ }^{b g}$ allele (Fig. 2B).

In this study, we designed several primers for the genetic analysis of the Lyst locus [4]. None of the primers designed on the L1 sequences amplified the DNA fragments specific to the mutant alleles because L1 is highly repeated and dispersed in the mammalian
(A)

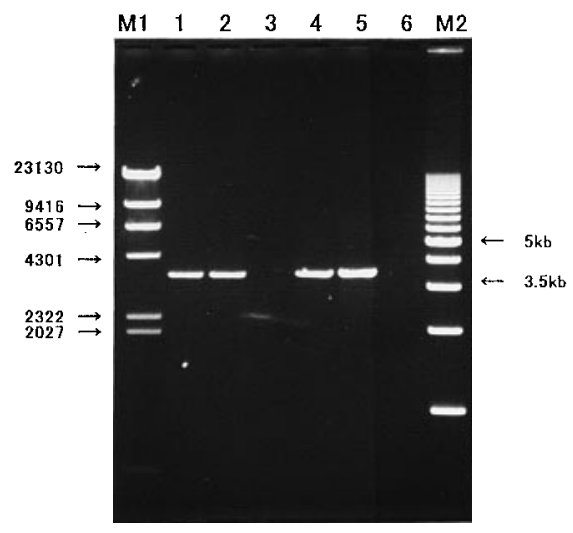

(B)

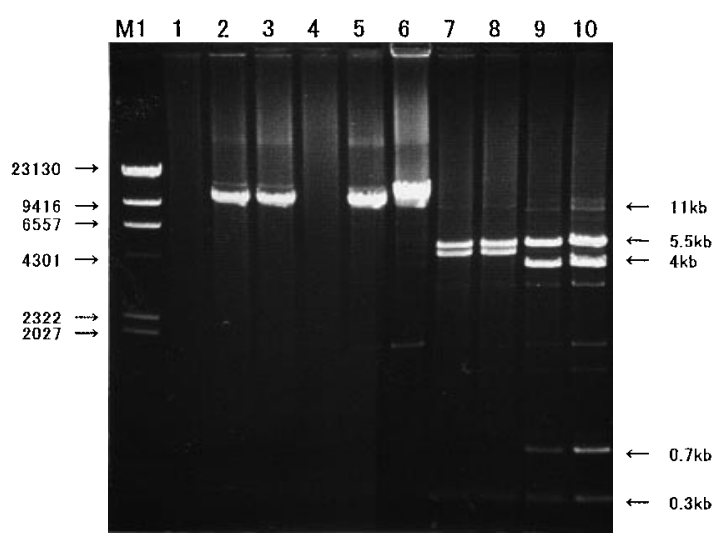

Fig. 2. Electrophoretic patterns obtained by nested PCR-RFLP analysis for discriminating rat $L y s t$ alleles. (A) PCR products obtained by the $\mathrm{Bg} 41 \mathrm{C} / / \mathrm{Bg} 50$ primer pair. Lane 1: ACI/N. Lane 2: ACI/N-Lyst $t^{b g-K y o} /+$ heterozygote. Lane 3: ACI/N-Lyst $t^{b g-K y o} / L y s t^{b g-K y o}$ homozygote. Lane 4: DA/ Slc. Lane 5: DA-Lyst $t^{b /} /+$ heterozygote. Lane 6: DALyst $t^{b g} / L y s t^{b g}$ homozygote. (B) Nested PCR products of the Bg39A4//Bg52A4 and Bg39A4nes//Bg52A4nes primer pairs (Lane 1-6) and the nested PCR products digested with StuI (Lane 7-10). Lane 1: ACI/N. Lane 2, 7: ACI/N-Lyst ${ }^{b g-K y o} /+$ heterozygote. Lane 3, 8: ACI/N-

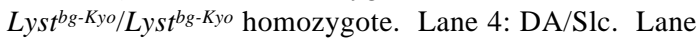
5, 9: DA-Lyst $t^{b g} /+$ heterozygote. Lane 6, 10: DA-Lyst $t^{b g} /$ Lys $t^{b g}$ homozygote. Lane M1: Lambda Hind III DNA size marker. Lane M2: 1kb DNA Ladder size marker.

genome [6]. A Bg41C//Bg50 primer pair amplified the specific PCR product only in the normal Lyst allele, since the $\mathrm{Bg} 41 \mathrm{C}$ primer sequence designed on exon 28 and the Bg50 primer sequence on exon 29 are in the deleted regions of both the mutant alleles. However, the primer pairs for the mutant alleles did not amplify 
the targeted PCR products in the normal Lyst allele owing to the long size of the fragment sequence. The position of the Bg39A4 primer is over $30 \mathrm{~kb}$ distant from the position of the $\mathrm{Bg} 52 \mathrm{~A} 4$ primer in the genomic sequence of the non-deleted normal Lyst allele which is the same as that of the $\mathrm{Bg} 39 \mathrm{~A} 4 \mathrm{nes} / \mathrm{Bg} 52 \mathrm{~A} 4$ nes inner primer pair.

The PCR analysis using the $\mathrm{Bg} 39 \mathrm{~A} 4 / / \mathrm{Bg} 52 \mathrm{~A} 4$ primer pair did not show sufficient sensitivity and reproducibility for the detection of the mutant alleles in the Lyst ${ }^{b g-K y o} /+$ and the Lyst $^{b g} /+$ heterozygotes. However, nested PCR analysis made it possible to detect both the beige mutant alleles in the Lyst locus with high sensitivity, reproducibility and specificity. Moreover, combining RFLP with StuI digestion made it possible to accurately discriminate among the three alleles on the Lyst locus. The nested PCR-RFLP analysis used in this study will be useful for developing and maintaining congenic strains with the Lyst ${ }^{b g}$ gene and/or the Lyst ${ }^{\text {bg-Kyo }}$ gene.

\section{References}

1. Baetz, K., Isaaz, S., and Griffiths, G.M. 1995. J. Immunol. 154: 6122-6131.
2. Beguez-Cesar, A. 1943. Bol. Soc. Cubana Pediatr. 15: 900922.

3. Blume, R.S. and Wolff, S.M. 1972. Med. Baltimore 51: 247-280.

4. Casavant, N.C. and Hardies, S.C. 1993. Mamm. Genome 4: 193-201.

5. Clark, R. and Kimball, H. 1971. J. Clin. Invest. 50: 26452652.

6. Dorner, M. and Paabo, S. 1995. Mol. Biol. Evol. 12: 944948.

7. Haliotis, T., Roder, J., Klein, M., Ortaldo, J., Fauci, A.S., and Herberman, R.B. 1980. J. Exp. Med. 151: 1039-1048.

8. Masui, N., Nishikawa, T., Takagi, Y., Mori, M., Suzuki, T., and Sato, K. 2003. Exp. Anim. 52: 89-91.

9. Mori, M., Nishikawa, T., Higuchi, K., and Nishimura, M. 1999. Mamm. Genome 10: 692-695.

10. Mori, M., Yamasaki, K., Nanishi, S., Kitada, K., Higuchi, K., Namiki, C., Hamada, S., and Serikawa, T. 2003. Exp. Anim. 52: 31-36.

11. NCBI Web. Site; http//w.w.w.ncbi.nlm.nih.gov/Genbank/ index.html

12. Nishikawa, T. and Nishimura, M. 2000. Exp. Anim. 49: 43-45.

13. Nishimura, M., Inoue, M., Nakano, T., Nishikawa, T., Miyamoto, M., Kobayashi, T., and Kitamura, Y. 1989. Blood 74: 270-273.

14. Sato, A. 1955. Tohoku J. Exp. Med. 61: 201.

15. Targan, S. and Oseas, R. 1983. J. Immunol. 130: 26712674.

16. The rat genome database, http://ratmap.gen.gu.se 